transfer of a recombinant $\alpha_{1}$-antitrypsin gene to the lung epithelium in vivo. Science 1991; 252: 431-4.

22 Strauss WM, Jaenisch R. Molecular complementation of a collagen mutation in mamn

23 Stewart MJ, Plautz GE, Del Bouno L, et al. Gene transfer in vivo with DNAliposome complexes: safety and acute toxicity in mice. Human Gene Therapy 1992; 3: 267-76.

24 Miller AD. Human gene therapy comes of age. Nature $1992 ; 357: 455-60$.

25 Nabel GJ, Chang A, Nabel EG, et al. Clinical protocol; immunotherapy of malignancy by in vivo gene transfer into tumors. Human Gene Therapy 1992; 3: $399-410$.

26 Curiel DT, Wagner E, Cotten M, et al. High-efficiency gene transfer mediated by adenovirus coupled to DNA-polylysine complexes. Human Gene Therapy 1992; 3: 147-54.

27 Wagner E, Zatloukal K, Cotten M, et al. Coupling of adenovirus to transferrinpolylysine/DNA complexes greatly enhances receptor-mediated gene delivery and expression of transfected genes. Proc Natl Acad Sci USA 1992; 89: 6099-103.

28 Wang C-Y, Huang L. pH-sensitive immunoliposomes mediate target-cellspecific delivery and controlled expression of a foreign gene in mouse. Proc specific delivery and controlled expre

29 Cotten M, Wagner E, Zatioukal K, et al. High-efficiency receptor-mediated delivery of small and large 48 kilobase gene constructs using the endosomedisruption activity of defective or chemically inactivated adenovirus particles. Proc Natl Acad Sci USA 1992; 89: 6094-8.

\title{
Haemophilus influenzae type b
}

The addition of a vaccine for infants against Haemophilus influenzae type $b$ is among several significant changes made in recent years to the routine schedule of immunisation in the UK and the Republic of Ireland. In Western countries $H$ influenzae type $\mathrm{b}$ has been the most common cause of bacterial meningitis and acute epiglottitis in early childhood and also a leading cause of pneumonia, septic arthritis, and cellulitis.

Since 1 October 1992, children $<4$ years of age in the UK and the Republic of Ireland have been eligible for immunisation against $H$ influenzae type $b$. Two conjugate vaccines are in use: PRP-T (Pasteur-Merieux, a conjugate of the purified polysaccharide, polyribosylribitol phosphate (PRP) linked covalently to tetanus toxoid) and HbOC (Praxis-Lederle, sized oligosaccharides derived from PRP conjugated to a non-toxic variant of diphtheria toxin, $\left.C_{R M} M_{197}\right)$. Both were shown to be highly protective in efficacy studies. ${ }^{12}$ Furthermore, there is evidence from Finland that implementation of a vaccination programme in the community could go close to eradicating $H$ influenzae type b disease. ${ }^{3}$ However another $H$ influenzae type b vaccine PRP-D (a conjugate of PRP and diphtheria toxoid), which was highly efficacious in Finland, was poorly protective when studied in a different ethnic group (Alaskan Eskimos) ${ }^{4}$

Several important questions remain to be answered.

(1) How well will $H$ influenzae type b conjugate vaccines perform in the field in the British Isles?

(2) How can their efficacy be measured?

(3) Is there any increase in the incidence of $H$ influenzae type $b$ infection in the period from vaccination until immunity has developed? (In infants $<13$ months, they are incompletely immunised until 1 week after at least two, and preferably three, doses have been given. In an older child, 2-3 weeks must elapse before protection develops after a single dose.)

(4) Does invasive infection that occurs despite 'incomplete' vaccination result in immunity?

(5) Is a booster dose of vaccine required in the second year of life?

A special opportunity now exists to address these questions through a British Paediatric Surveillance Unit (BPSU) study of invasive $H$ influenzae type b disease occurring after vaccination against $H$ influenzae type b. Contrary to the practice in the USA and mainland Europe, the UK and the Republic of Ireland offer only primary immunisation against $H$ influenzae type b with no booster dose in the second year of life. This is despite the fact that primary immunisation is now completed at a much younger age (4 months in the UK and 6 months in the Republic of Ireland), another recent and significant change to the routine immunisation schedule. Conjugate vaccines induce $T$ cell dependent memory and thus there is the potential for a booster response should the $H$ influenzae type $\mathrm{b}$ organism or cross reactive antigen be encountered subsequently. There are at present no strong epidemiological data to support the need for a booster dose. It is clearly vital that cases of invasive $H$ influenzae type $b$ infection in children who have been appropriately immunised are notified. Complete reporting of such 'true' vaccine failures will enable a meaningful audit of vaccine efficacy and determine if waning immunity (after primary immunisation has been completed) can result in children becoming susceptible to invasive $H$ influenzae type b disease.

Auditing of interventions that purport to promote health is becoming an integral part of modern medical practice. Postmarketing surveillance through the BPSU study in the British Isles offers several distinct advantages over that being performed elsewhere in the world (for example, in the USA where various $H$ influenzae type $\mathrm{b}$ conjugate vaccines have been offered since 1987 in a piecemeal and evolving way).

These advantages include the following:

(1) Definition of the target population is a straightforward task because of the defined date (1 October 1992) for beginning vaccination and the clear guidelines given as to who is eligible (children $<4$ years).

(2) The vaccination status of any particular child in the UK can usually be easily ascertained by reference to district computer records with only occasional recourse required to parent held, general practitioner, or health visitor records.

(3) The childhood immunisation programme includes a standardised approach across the UK that is centrally organised but also involves the provision of excellent peripheral support through the work of immunisation coordinators (health professionals specifically designated to advise on and promote immunisation) in each health district.

(4) A guide for UK doctors, Immunisation against Infectious Disease, is widely distributed and regularly updated. ${ }^{5}$

(5) There is excellent professional and consumer confidence in the childhood immunisation programme and very high rates of immunisation uptake ( $90 \%$ and greater) are now being achieved in most districts of the UK. The rates are lower in the Republic of Ireland.

(6) In the UK and the Republic of Ireland, infants $<13$ months of age are only being offered one $H$ influenzae type $b$ conjugate vaccine (PRP-T in the UK and $\mathrm{HbOC}$ in the Republic of Ireland) so any impact on disease in this age group by a particular vaccine is easier to analyse. Older UK children (between 1 and 4 years) are offered one injection with either one of the two vaccines PRP-T and HbOC whereas only $\mathrm{HbOC}$ is offered in the Republic of Ireland.

(7) The sheer scale of the childhood immunisation programme in the British Isles (more than 3 million children aged less than 4 years of age) means that important questions 
about vaccine efficacy and immunity can be answered quickly providing there is continued enthusiastic cooperation from paediatricians in BPSU studies such as the study of invasive $H$ influenzae type $\mathrm{b}$ infection in vaccinated children. *

These questions relating to $H$ influenzae type $\mathrm{b}$ vaccine serve to exemplify a more general issue. They highlight the importance of adequate audit of changes to public health policy (such as immunisation practices), rigorous attention to which might lessen or circumvent unnecessary controversy such as the recent precipitous withdrawal of two measles, mumps and rubella vaccines from routine supply which occurred in the UK.

*Cases should be notified by phone (day or night) to Dr M Slack, Haemophilus Reference Laboratory, John Radcliffe Hospital, Oxford (telephone 0865 220852). In the Republic of Ireland, cases should be notified to Dr J Fogarty, Hib Study, Western Health Board, Galway (telephone 091-23122)
We acknowledge the support of the Medical Research Fund of Children Nationwide for their support of the BPSU.

R BOOY

ER MOXON

Department of Paediatrics,

Fohn Radcliffe Hospital,

Headington,

Oxford OX3 9DU

1 Booy R, Moxon ER, Macfarlane JA, Mayon-White RT, Slack MPE. Efficacy of Haemophilus influenzae type $b$ conjugate vaccine in Oxford region. Lance 1992; ii: 847

2 Black SB, Shinefield HR, Fireman B, et al. Efficacy in infancy of oligosaccharide conjugate Haemophilus influenzae type b (HbOC) vaccine in a United States population of 61,080 children. Pediatr Infect Dis $\mathcal{F} 1991 ; 10: 97-104$.

3 Peltola H, Kilpi T, Anttila M. Rapid disappearance of Haemophilus influenzae type b meningitis after routine childhood immunisation with conjugate vaccines. Lancet 1992; ii: 592-4.

4 Ward J, Brenneman G, Letson GW, Heyward WL. Limited efficacy of Haemophilus influenzae type $b$ conjugate vaccine in Alaska native infants. NEngl f Med 1990; 323: 1393-401.

5 Department of Health. Immunisation against infectious disease. London: HMSO, 1992.

\section{How to get drugs into the respiratory tract}

Virtually any drug solution or suspension may be delivered to the respiratory tract as an aerosol. This opens a wide range of options for direct treatment of respiratory diseases and provides a port of entry for systemic drug treatment.

In childhood, drug doses have largely evolved empirically and aerosol delivery methods have been adapted from adult practice. However, problems are encountered in getting drugs into the respiratory tract of young children. These include compliance, anatomical and physiological variations due to age, problems with drug delivery, and drug delivery devices.

\section{Compliance}

Compliance with treatment is, and will probably remain, the main difficulty in getting aerosols into the airways of children. It is essential to ensure that the parents and child understand the need for treatment, and to give careful, patient, and repeated explanation of inhaler techniques with positive reinforcement.

\section{Anatomical and physiological problems}

Although the majority of young children nose breathe at rest, the mode of children's breathing under different circumstances is not known. Nasal breathing is associated with lower lung deposition of aerosolised drugs in adults, but little is known about this in children. The absence of nasal hair in the preadolescent and the fact that the infant's head is larger with respect to its body than in adulthood may make nasal breathing less of a problem than would be expected.

Young children usually breathe tidally when given aerosolised medications. This may reduce deposition of drug in the lung peripheries compared with that of older, compliant children who inhale deeply and slowly.

Recent work focusing on the amount of nebulised drug available for inhalation demonstrates that the quantity of nebulised aerosol inspired, which includes that deposited in the nose and upper airways, may be independent of the size of the child after 6 months of age. ${ }^{1}$ Infants younger than this have small tidal volumes and inspiratory flow rates, and therefore inspire aerosol directly from the nebuliser without entraining surrounding room air. As the child grows and tidal volume increases, a greater amount of surrounding air not containing aerosol is inspired (entrained air). Thus the concentration of aerosol per breath of an older child is less than that of the younger child. The total dose inhaled may be similar, but dose per kilogram will be much less in the older child. It has been suggested that, on the basis of these findings, aerosols should be administered on a weight corrected concentration basis for children aged more than 6 months.

Indeed the age related decline in airways responsiveness reported in recent studies might reflect failure to correct adequately the test dose for the child's size rather than a genuine physiological event. ${ }^{2}$ Many hospitals, however, use a standard dosage for all ages in the case of antiasthma drugs, such as sodium cromoglycate and $\beta_{2}$ agonists, where side effects are uncommon.

\section{Drug or delivery failure?}

It is now well recognised that the disappointing clinical effect of some aerosolised drugs may be simply due to inadequate amounts reaching the airways.

This is illustrated by the initial use of nebulised steroids in young asthmatics. Beclomethasone dipropionate, delivered by spacer devices or powered capsule, usually results in substantial symptomatic improvement in asthmatic children. ${ }^{3}$ However, when administered by nebulisation, clinical response was disappointing, and the value of nebulised steroids in young asthmatics was questioned. ${ }^{4}$ Beclomethasone dipropionate is nebulised as a suspension, due to its poor solubility. Steroid particles leaving the nebuliser are surrounded by a layer of fluid, increasing their size and explaining the poor clinical effect. For the same initial dose of beclomethasone dipropionate given by a spacer device a much greater amount (5-10 fold) of drug is contained in particles of less than five microns diameter, the size below which lung deposition is likely. ${ }^{5}$ As anticipated from these results, use of spacer devices with a facemask attachment to deliver steroid aerosols to very young children and infants has shown an excellent clinical response in many cases. ${ }^{6}$ 